\title{
Mechanisms of Protein and Virus Crystal Growth; an Atomic Force Microscopy Study of Canavalin and STMV
}

\author{
T. A. Land, A. J. Malkin, Y. G. Kutznesov \\ A. McPherson, J. J. DeYoreo
}

This paper was prepared for submittal to

The 11th International Conference on Crystal Growth

The Hague, The Netherlands

June 1995

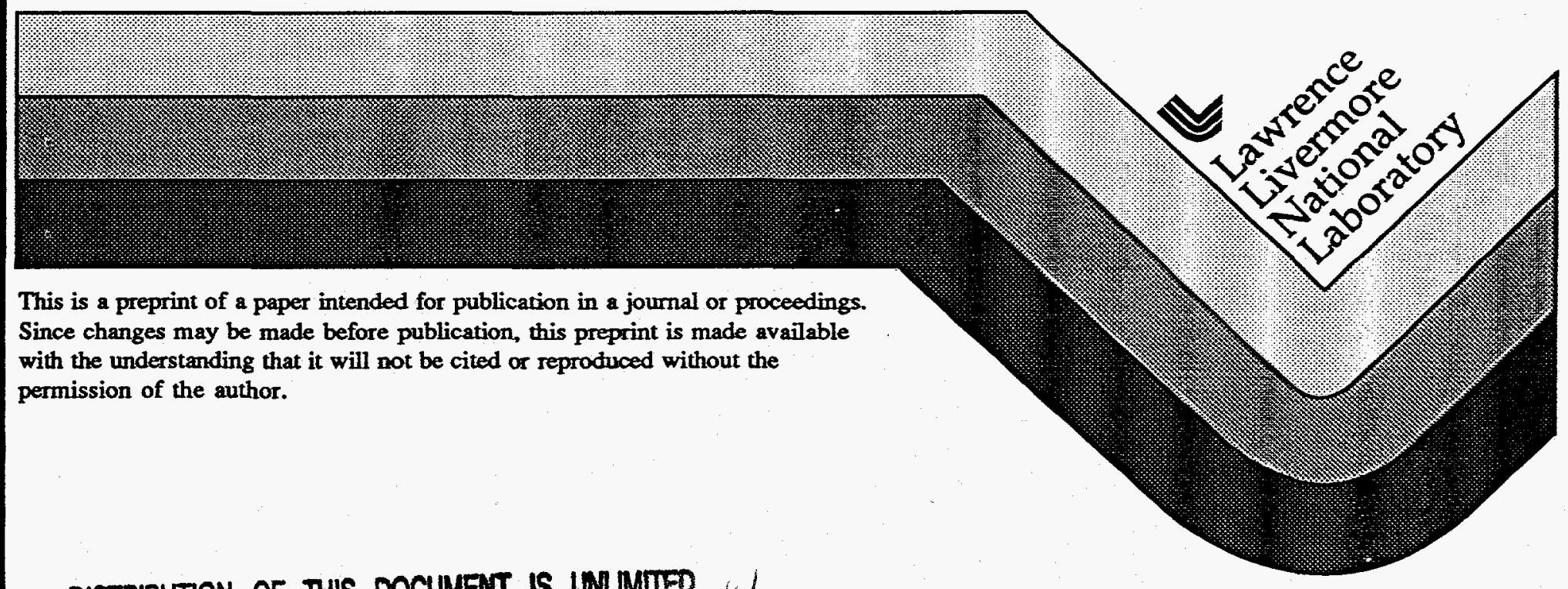

DISTRBUTION OF THIS DOCUMEAT IS UNLMMTED WW 


\section{DISCLAIMER}

Portions of this document may be illegible in electronic image products. Images are produced from the best available original document. 


\title{
Accepted to the Journal of Crystal Growth Sept. 1995
}

\section{Mechanisms of protein and virus crystal growth: An atomic force microscopy study of canavalin and STMV crystallization}

\author{
T.A. Land, A.J. Malkin*, Yu.G. Kutznesov*, A. McPherson*, and J.J De Yoreo \\ Department of Chemistry and Materials Science, Lawrence Livermore National Laboratory, \\ Livermore, CA 94550 \\ *Department of Biochemistry, University of California at Riverside, Riverside, CA, 92521
}

\section{abstract}

The evolution of surface morphology and step dynamics during growth of rhombohedral crystals of the protein canavalin and crystals of the cubic satellite tobacco mosaic virus (STMV) have been investigated for the first time by in situ atomic force microscopy. These two crystals were observed to grow by very different mechanisms. Growth of canavalin occurs on complex vicinal hillocks formed by multiple, independently acting screw dislocations. Small clusters were observed on the terraces. STMV on the other hand, was observed to grow by $2 \mathrm{D}$ nucleation of islands. No dislocations were found on the crystal. The results are used to determine the growth mechanisms and estimate the fundamental materials parameters. The images also illustrate the important mechanism of defect incorporation and provide insight to the processes that limit the growth rate and uniformity of these crystals.

\section{Introduction}

The crucial functions of biological systems are governed and mediated by nucleic acids and proteins, as well as complexes of the two. The specific roles of the macromolecules are in turn defined by their detailed structures that are determined using x-ray crystallography. ${ }^{1}$ To apply this technique, it is essential to grow large, relatively perfect, single crystals of the macromolecules. However, crystallization of proteins and other biological macromolecules is highly problematic, to such an extent in fact that it has become the rate-limiting step in most accurate structure analysis. This is true, in large part, because little is known of the mechanisms and forces that promote or inhibit protein crystal growth, and virtually nothing is known of the fundamental thermodynamic and kinetic parameters that underlie macromolecular crystallization. ${ }^{1}$ Little is known, for 
example, of the role of transport processes in protein crystal growth. Still less is understood of the events leading to nucleation, of the mechanisms by which crystals grow, how defects are incorporated, or of the forces responsible for the orientation and bonding of the molecules in the lattice. A more detailed and comprehensive understanding of all of these questions are required if the current obstacles to macromolecular crystal growth are to be overcome and the full potential of $\mathrm{x}$-ray crystallography to molecular biology and medicine realized.

Here, we report the first results results of in situ atomic force microscopy on single crystals of the protein canavalin and the virus STMV that reveal two different modes of growth. The results show that growth of canavalin crystals occurs on complex vicinal hillocks formed by multiple, independently acting screw dislocation sources. On the terraces of these steps we observe the continual formation of a large number of molecular clusters with diameters of less than ten molecules. These clusters are mobile and become incorporated at step edges as the steps move across the terraces. Finally, we show that the growth rate of canavalin is enhanced in the region scanned by the AFM tip, showing that, under typical conditions, canavalin crystal growth is bulk-diffusion limited. In contrast to the dislocation growth mechanism of canavalin, STMV was observed to grow by $2 \mathrm{D}$ nucleation. The $2 \mathrm{D}$ islands expanded to form a "stack" that eventually covered the entire crystal. Growth of STMV does not appear to be limited by volume diffusion, as was canavalin, but rather by the kinetics of monomer incorporation. Fundamental growth parameters were determined for both materials. From the size of the critical radius the free energy per-unit-step height of the step edge, $\alpha$, is estimated, and from the speed of the steps, the kinetic coefficient for step motion, $\beta$, is calculated.

\section{Experimental}

Stock aqueous solutions of canavalin with a concentration of $38 \mathrm{mg} / \mathrm{ml}$ were prepared from defatted jack bean meal as described previously. ${ }^{2}$ Crystals of canavalin were nucleated on etched glass substrates within the sealed fluid cell of a commercial atomic force microscope whose basic operation has been described elsewhere. ${ }^{3}$ After mixing of the stock solution with the precipitant 
Dullecco-Phosphate buffered saline (2DPBS) in the ratio of 1:1.5, the resulting solution was injected into the cell and allowed to incubate until crystals on the order of $50 \mu \mathrm{m}$ in size were observed. STMV crystals were nucleated in the same manner by incubation of a $2.3 \mathrm{mg} / \mathrm{ml}$ STMV solution with an equal volume of $40 \%$ saturated ammonium sulfate. Images were collected in the contact mode using a cantilever with a nominal force constant of $0.38 \mathrm{Nm}^{-1}$. During the course of the experiments a constant effort was made to minimize the force.

\section{Results: Canavalin}

Canavalin crystallizes in many forms depending on the crystallization conditions. ${ }^{4} \mathrm{We}$ observed the growth on the $\{100\}$ face of rhombohedral canavalin crystals of space group R3. The canavalin trimer forms a three-sided ring with an outside diameter of 8.6-8.8 $\mathrm{nm}$ and a thickness of $3.5-4.0 \mathrm{~nm}$. The unit cell dimensions are $a=b=c=8.3 \mathrm{~nm}$ with $\gamma=111.1^{\circ}$, and the resulting distance between the $\{100\}$ planes is $7.2 \mathrm{~nm}$.

A high-resolution image of the canavalin surface is shown in Fig. 1. The lower portion has been filtered using the Fourier transform of the image for clarity. The rhombohedral structure is clear and the measured lattice spacing and angles agree with the crystallographic data for this structure. ${ }^{4}$

Growth of canavalin crystals was observed to occur by a dislocation mechanism. Fig.2 is a series of sequential $23 \mu \mathrm{m}$ images showing the evolution of a dislocation hillock on a $50 \mu \mathrm{m}$ crystal. This hillock was the only growth source on the crystal and consists of seven separate dislocation sources generating a total of eight steps. Each source acts independently of the others in generating steps that merge to form the overall growth source, and no single dislocation source is dominant.

The terraces in these images contain many small clusters. Fig.3 is a series of sequential $1 \mathrm{x} 1 \mu \mathrm{m}$ images that show how these clusters interact with the advancing step and how the cluster distribution evolves with time. While some of the clusters desorb or dissolve on the time scale of imaging, most are stable and have low mobility. The clusters are incorporated into the advancing 
steps and thus provide a significant fraction of the molecules in the crystal. Little or no coalescence of adjacent clusters is observed nor do the clusters grow with time. In fact, the clusters are of nearly uniform size measuring $70-90 \mathrm{~nm}$ and $40-60 \mathrm{~nm}$ in the fast and slow scan directions respectively and $3.9 \mathrm{~nm}$ in height. While distortion in the fast scan direction is likely for weakly bound objects on the surface, distortion in the slow scan direction should be considerably less. However, the small size of the clusters precludes resolution of their internal structure, and we can neither distinguish between amorphous and ordered packing nor determine whether the cluster subunits are monomers, trimers, or other oligimers. If these clusters are ordered, then the size range in this direction is consistent with that of a hexagonally close-packed cluster of canavalin trimers with the first few rings filled. We suggest that these clusters may have a stable configuration of canavalin molecules and that further additions lead to weakly bound molecules which are energetically unfavorable at these supersaturations.

The curvature of outgrowths, or capes, on the steps can be used to estimate $\alpha$, the free energy of the step edge per-unit-step height. The relationship between $\alpha$ and $\mathbf{r}_{\mathbf{c}}$, the critical radius of curvature for a step is given by: ${ }^{5,6}$

$$
r_{c}=\omega \alpha / k T \sigma
$$

where $\omega$ is the specific molecular volume and is equal to $4.1 \times 10^{-19} \mathrm{~cm}^{3}$ in rhombohedral canavalin, $\mathrm{k}$ is Boltzman's constant, $\mathrm{T}$ is the temperature, and $\sigma=\ln \left(\mathrm{C} / \mathrm{C}_{\mathrm{e}}\right)=0.6$ is the relative supersaturation where $\mathrm{C}=1.2 \times 10^{16} \mathrm{~cm}^{3}$ and $\mathrm{C}_{\mathrm{e}}=0.65 \times 10^{16} \mathrm{~cm}^{3}$ are the actual and equilibrium volume concentrations of canavalin in solution. The maximum curvature of the steps, based on a large number of measurements of small capes on the step edges, is $95 \pm 28 \mathrm{~nm}$. For the supersaturation used in the experiments, and using a value of $95 \mathrm{~nm}$ for $\mathrm{r}_{\mathrm{c}}$, we find that $\alpha<0.6$ $\mathrm{erg} / \mathrm{cm}^{2}$. 
The step velocity, $v$, can be used to calculate the kinetic coefficient, $\beta$, that is defined by the relationship: $: 5$

$$
v=\omega \beta\left(C-C_{e}\right)
$$

The kinetic coefficient itself, is a measure of the kinetics of adsorption, diffusion, and incorporation with the rate-limiting step dominating the value of $\beta$. A typical value of $\beta$ for inorganic crystals such as $\mathrm{KH}_{2} \mathrm{PO}_{4}$ is on the order of $10^{-1}-10^{-2} \mathrm{~cm} \cdot \mathrm{s}^{-1}$. For canavalin we calculate a kinetic coefficient in the range of $\sim 10^{-5}$ to $10^{-4} \mathrm{~cm} \bullet \mathrm{s}^{-1}$. This value of $\beta$ is similar to that found for crystals of the satellite bobacco mosaic virus as discussed in the next section. ${ }^{7}$ Thus the kinetic coefficient of elementary steps is about three orders of magnitude smaller in macromolecular biological crystals than in inorganic crystals. The potential sources for the low value of $\beta$ in these organic systems include: a large barrier to adsorption (shedding the hydration layer), low surface diffusivity, and a low probability that an incoming molecule has the proper orientation for incorporation into the crystal.

Finally, we found that the growth rate of canavalin was enhanced in the area scanned by the AFM tip. Upon increasing the scan size, the steps which had been generated within the scanned region were found to be "bunched up" at the edges as shown in Fig. 2f. This observation suggest that the tip may act to break up the boundary layer at the crystal surface and enhance the mixing of the solution thereby increasing the growth rate. Thus, under typical conditions, canavalin crystallization appears to be diffusion limited.

\section{Results: STMV}

Results from this AFM study helped to determine the molecular packing of cubic STMV crystals. The icosohedral virus is essentially spherical in shape with a diameter of $17 \mathrm{~nm} .^{8} \mathrm{X}$-ray diffraction has been used to determine a $\mathrm{P} 23$ space group with $\mathrm{a}=\mathrm{b}=\mathrm{c}=25.7 \mathrm{~nm}$ for the cubic crystal form, however, this crystal class does not reflect fcc packing due to the fact that the STMV molecule itself lacks 4-fold symmetry. A high-resolution AFM image of the lattice is shown in 
Fig. 4. Images such as this were used to determine that the (111) plane consist of a hexagonally close packed arrangement of the molecules. The molecular spacings and step heights are consistent with those expected for an fcc unit cell..$^{8,9,10}$ This data, combined with the x-ray data, suggest that STMV forms an fcc crystal with P23 symmetry.

Growth of the STMV crystals occurred by two-dimensional nucleation and layer by layer advancement of monomolecular steps of $14 \mathrm{~nm}$ as shown in Fig. 5. Dislocations are typically thought of as the primary growth mechanism ${ }^{5,6}$. Surprisingly, no dislocations were observed on the entire $50 \mu \mathrm{m}$ STMV crystal. We found that the "stacks" were the growth centers for the crystal. The stack evolution is shown in Fig. 5. The $7.5 \mu \mathrm{m}$ image in Fig. 5a was taken early in the experiment (note that the micron size particle seen in Figs. 5b-f and Fig. 6 is absent here.) These stacks are formed by nucleation of new islands on the upper terraces. Once formed, these islands continue to grow outward in the plane and may coalesce with other islands on the same terrace as seen in Fig. 5d. The speed of the step edges on the islands begins to slow down as they catch up with the edge of the previous terrace resulting in "step bunching" and formation of a "stack". As seen in the images, the shape of the individual islands as well as the overall stack is circular. Finally, in Fig. $5 \mathrm{f}$ which was taken at a later time, the step train from another "stack" is seen in the lower right corner of the image.

For growth to occur by $2 \mathrm{D}$ nucleation, a stable nucleus with a radius $\geq r_{c}$ must first be formed. ${ }^{5,6}$ A critical nucleus represents a local-free-energy maximum where, for $\mathrm{r}<\mathrm{r}_{\mathrm{c}}$ dissolution occurs, while for $r>r_{c}$ growth may proceed, in both cases with a decrease in free energy. In our experiments, we observed small stable islands that continued to grow as well as small islands that dissolved. Measurements of these islands allowed us to bracket the size of the critical nucleus necessary for growth to occur to lie in the range from 100 to $300 \mathrm{~nm}$.

With knowledge of the critical radius size, the step-edge free energy can be determined using formula 1 , where $\omega=4.2 \times 10^{-18} \mathrm{~cm}^{3}, C=5.6 \times 10^{13} \mathrm{~cm}^{3}, C_{e}=3.4 \times 10^{14} \mathrm{~cm}^{3}$ and $\sigma=$ 1.8. The step-edge free energy, $\alpha$, was then calculated to be in the range of 0.17 to $0.34 \mathrm{erg} / \mathrm{cm}^{2}$. 
A similar value of $2 \times 10^{-2} \mathrm{erg} / \mathrm{cm}^{2}$ was previously estimated by inelastic light scattering. ${ }^{10}$ (While the value for $3 \mathrm{D}$ nucleation is also small, it is not necessarily the same quantity as the $\alpha$ of $2 \mathrm{D}$ nucleation.) The step free energy for canavalin and STMV are substantially lower than found for conventional inorganic crystals grown from solution. ${ }^{6}$ However, because the molecules are so large, the value of $\alpha \mathrm{A} / \mathrm{kT}$ where $\mathrm{A}$ is the surface area per lattice site is still much greater than one, and thus, the terraces should be atomicly flat as observed in this study. ${ }^{6}$ The low value of $\alpha$ undoubtedly reflects the high solvent content (30-90\%) of macromolecular crystals. ${ }^{10}$ Due to the nearly equivalent environments in solution and in the crystal, the amount of work required to create a surface unit of the crystal might well be expected lower. The low value for $\alpha$ may also be a consequence of the scarcity and marginal strength of intermolecular interactions per lattice unit in macromolecular crystals.

Another important fundamental material parameter is the kinetic coefficient $\beta$. Using formula 2 and measurements of the step velocity, $\beta$ was estimated to be (4-8) $\times 10^{-4} \mathrm{~cm} / \mathrm{sec}$. The step velocity was monitored from the formation of a new island to the point where "step bunching" occurred. As the terrace width began to shrink, i.e., the steps began to bunch, a decrease of less than $25 \%$ occurred in the step velocity. This observation, in light of the similar values for the mono and macro step rates, suggests that there is no significant overlap of the diffusion fields. The images shown in Figs. $5 \mathrm{f}$ and 6. show the step train from another "stack" that is sweeping over the area from the right side. This has important implications as it demonstrates that, unlike canavlin, the growth of the area being imaged is not enhanced relative to the rest of the crystal suggesting that the growth is not diffusion limited. Thus, we suggest, that cubic STMV crystal growth is limited by the kinetics of monomer incorporation, rather than by surface or volume diffusion.

A recurring question in macromolecular crystal growth is the nature and influence of incorporated impurities and the degree to which they affect the properties of the crystals. Numerous observations of impurity effects were made in this AFM study. One interesting example 
was the absorption of a micron-size particle on the crystal surface during the experiment and its subsequent effects. Fig. 6. shows the process of particle incorporation. Initially, the particle projected above the surface as seen in Figs. 5 and 6 a. In Fig. 6b, a step is just beginning to close around the particle. After the passing of $\sim 10$ steps the particle was covered and the area developed into a persistent micron-sized hole as steps then proceeded to "wrap around" the defect-incorporation site as seen in Fig. $6 c$. We further found that canavalin crystals contain defects in the form of hollow channels as large as $2 \mu \mathrm{m}$ in diameter. ${ }^{11}$ Some of these "holes" were the source regions for screw dislocations (see Fig. 2) demonstrating for the first time that, even in these macromolecular systems, the strain near screw dislocations leads to hollow dislocation cores as predicted ${ }^{12,13}$ and observed in inorganic systems. ${ }^{14}$ However, the diameters of these cores varied with time and between sources with equal Burgers vectors and, furthermore, a number of holes which we observed were not connected with screw dislocations and persisted for growth periods of many days.

\section{Summary}

These AFM experiments have provided considerable insight to the mechanisms that control the growth of canavalin and STMV crystals. Although both are macromolecular crystals, canavalin growth occurs on dislocation induced hillocks and is limited by volume diffusion, while the growth of STMV occurs by 2D nucleation and is limited by monomer incorporation. Fundamental growth parameters such as the step edge free energy $\alpha$ and the kinetic coefficient $\beta$ were determined for both materials. The subject of crystalline perfection and how defects are incorporated into a crystal are subjects of great importance. These experiments show the actual process of a defect being created as a large particle was incorporated into the STMV crystal generating a persistent micron-size hole at the incorporation site. 
This work was performed under the auspices of the division of Materials Science, U.S.

Department of Energy, and Lawrence Livermore National Laboratory, under contract No. W7405-ENG48 and was supported by a grant from NASA.

\section{Figure Legends:}

Figure 1: A $280 \times 280 \mathrm{~nm}$ AFM image of the canavalin lattice. The lower inset portion of the image has been Fourier filtered for clarity.

Figure 2: A series of AFM images showing the complex dislocation growth hillock on the canavalin surface. a-e) $23 \mu \mathrm{m}$ images taken at $86 \mathrm{sec}$ intervals. f) This $27 \mu \mathrm{m}$ image shows growth enhancement in the area that was previously imaged, see the raised, square portion of the image.

Figure 3: A series of three $1 \times 1 \mu \mathrm{m}$ images taken at $50.3 \mathrm{sec}$ intervals showing clusters on the terraces of the canavalin surface. Note clusters being incorporated into the step edge.

Figure 4: A $1 \times 1 \mu \mathrm{m}$ AFM image of the (111) plane of a cubic STMV crystal. The hexagonal array of the individual virus monomers is clearly shown. The lower inset portion of the image has been Fourier filtered for clarity.

Figure 5: STMV: 2D growth. A series of 6 AFM images showing the nucleation, growth, and coalescence of islands and the expansion of the "stack". a) A $7.5 \times 7.5 \mu \mathrm{m}$ image of the "stack" in its early stages $(t=0)$. Note that the micron-size particle seen in images $b-f$ is not present here. b-e) $25 \times 25 \mu \mathrm{m}$ images showing $2 \mathrm{D}$ nucleation, coalescence, and growth of the "stack" $(\mathrm{t}=$ $1500,1590,1670$, and $1840 \mathrm{sec})$. f) This $23 \times 23 \mu \mathrm{m}$ image was taken at a later time $(\mathrm{t}=3520$ sec). The approach of another "stack" can be seen in the lower right portion of the image. 
Figure 6: The micron-size particle seen in figure 4 being incorporated in the STMV crystal. a ) In this image, the particle is still several layers above the surface $(t=5,140 \mathrm{sec})$. In $(b)$, the layers have accumulated around it so that it extends only one monolayer above the surface $(t=5,440$ $\mathrm{sec})$. In (c), the particle has been incorporated into the growing crystal with the subsequent appearance of a near micron sized hole in the surface $(t=5,860 \mathrm{sec})$.

${ }^{1}$ C. Braden and J. Tooze, Introduction to Protien Science (Garland Publishing Inc., New York and London, 1991).

${ }^{2}$ S.C. Smith, S. Johnson, J. Andrews and A. McPherson, Plant Physiol. 70, 1199 (1982).

${ }^{3}$ A.J. Gratz, S. Manne, and P.K. Hansma, Science 251, 1343 (1991).

${ }^{4}$ T-P Ko, J.D. Ng, J. Day, A. Greenwood, and A. McPherson, Acta. Cryst. D49, 478.

${ }^{5}$ W.K. Burton, N. Cabrera, and F.C. Frank, Phil. Trans. Roy. Soc. London A , 243, 299 (1951).

${ }^{6}$ A.A. Chernov, Modern Crystallography III. Crystal Growth, vol. 36 (Springer Series, Berlin \& New York, 1984).

${ }^{7}$ A.J. Malkin, T.A. Land, Yu.G. Kuznetsov, A. McPherson, and J.J. DeYoreo, Phys. Rev Lett., accepted (1995).

${ }^{8}$ S. Koszelak, K. Leja, R. Cudney, and A. McPherson, Biophysical Journal, in press (1995).

${ }^{9}$ A.J. Malkin and A. McPherson, Acta Cryst. D50, 385-395 (1994).

${ }^{10}$ G.L. Gilliland, M. Tung, D.M. Blakeslee, and Ladner, Acta Cryst. D50, 408-413 (1994).

${ }^{11}$ T.A. Land, A.J. Malkin, Yu.G. Kuznetsov, A. McPherson, and J.J. DeYoreo, Phys. Rev. Lett., accepted (1995).

${ }^{12}$ F.C. Frank, Acta Cryst. 4, 497 (1951).

${ }^{13}$ 17. N. Cabrera and M.M. Levine, Phil. Mag. 1, 450 (1956).

${ }^{14}$ J.J. De Yoreo, T.A. Land, and B.J. Dair, Phys. Rev.Lett. 73, 838-841 (1994). 


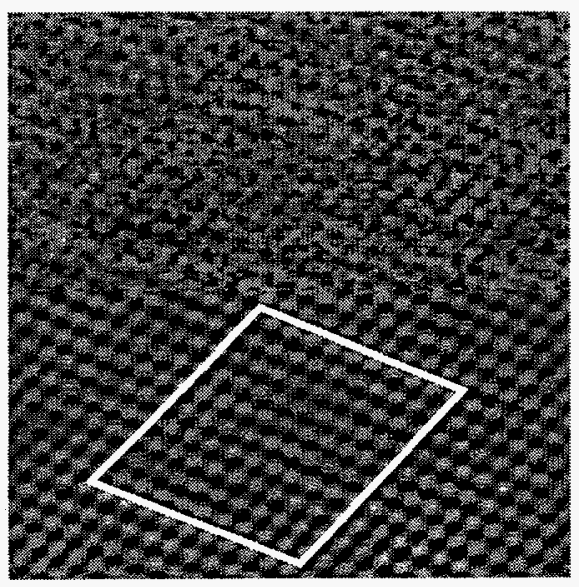

Figure 1 

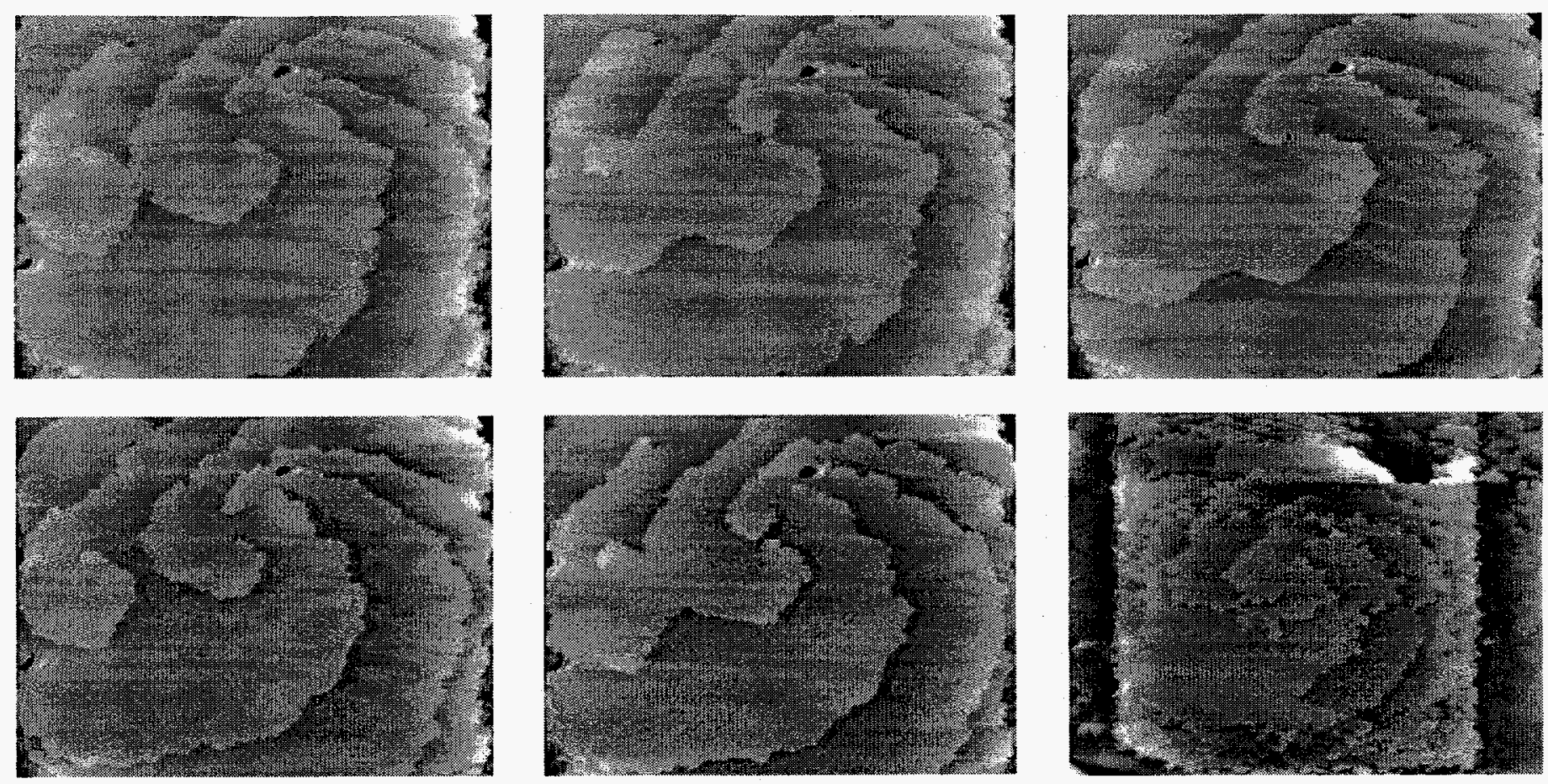

figure 2

DeYoreo 

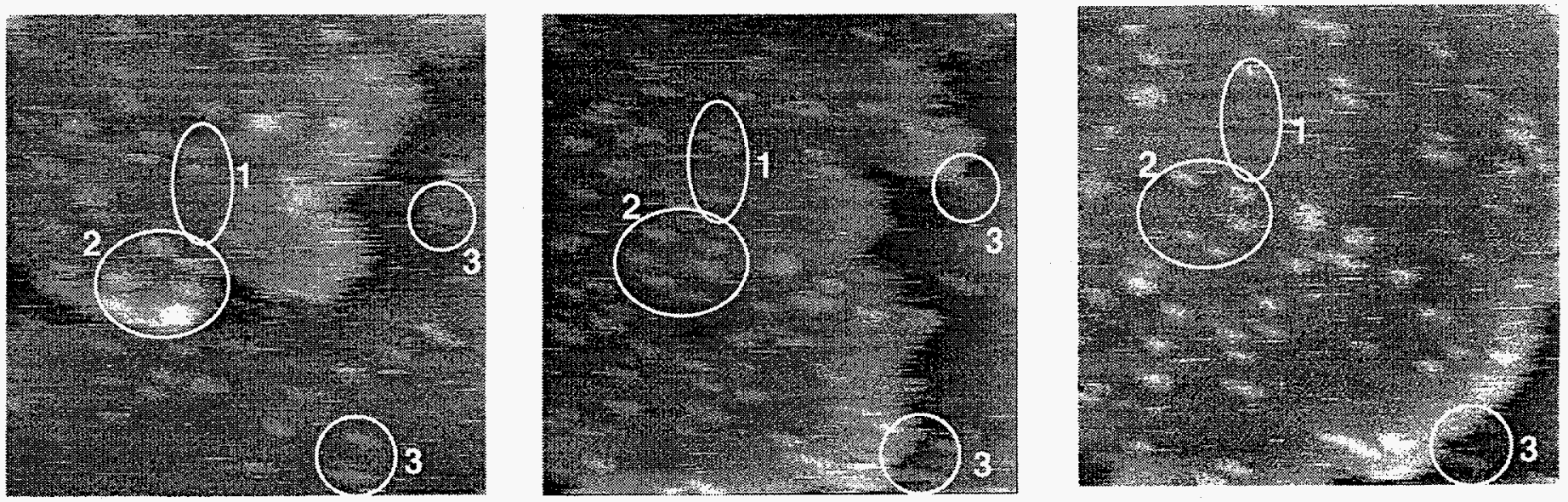

figure 3 


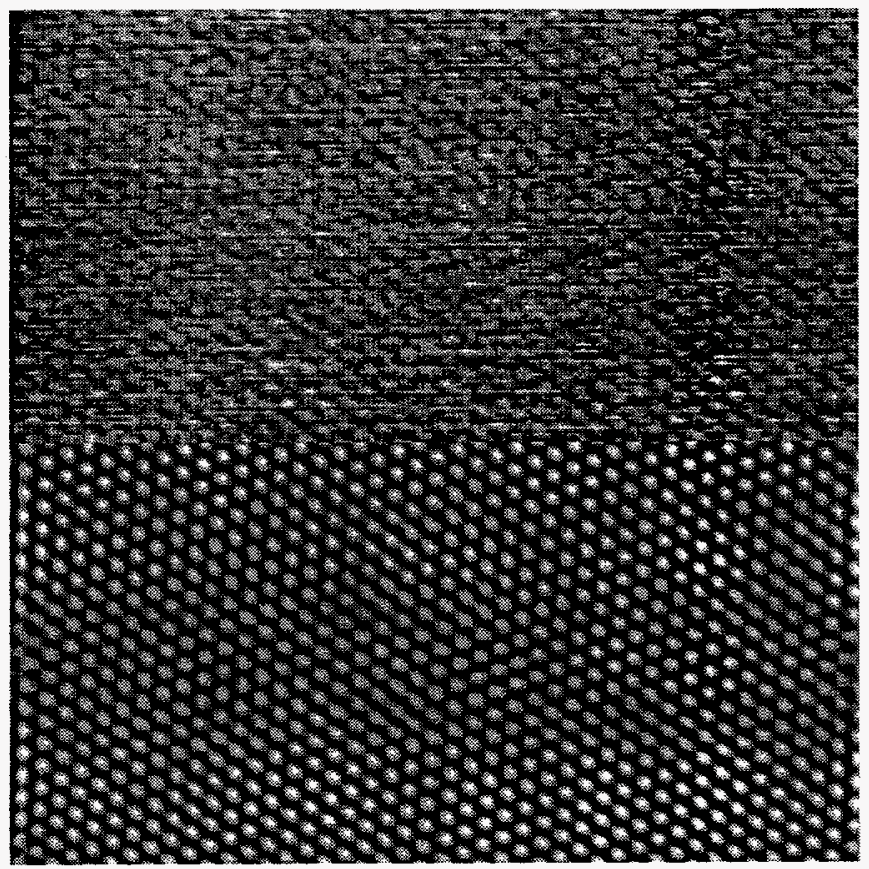

figure 4

DeYoreo, STMV Science 

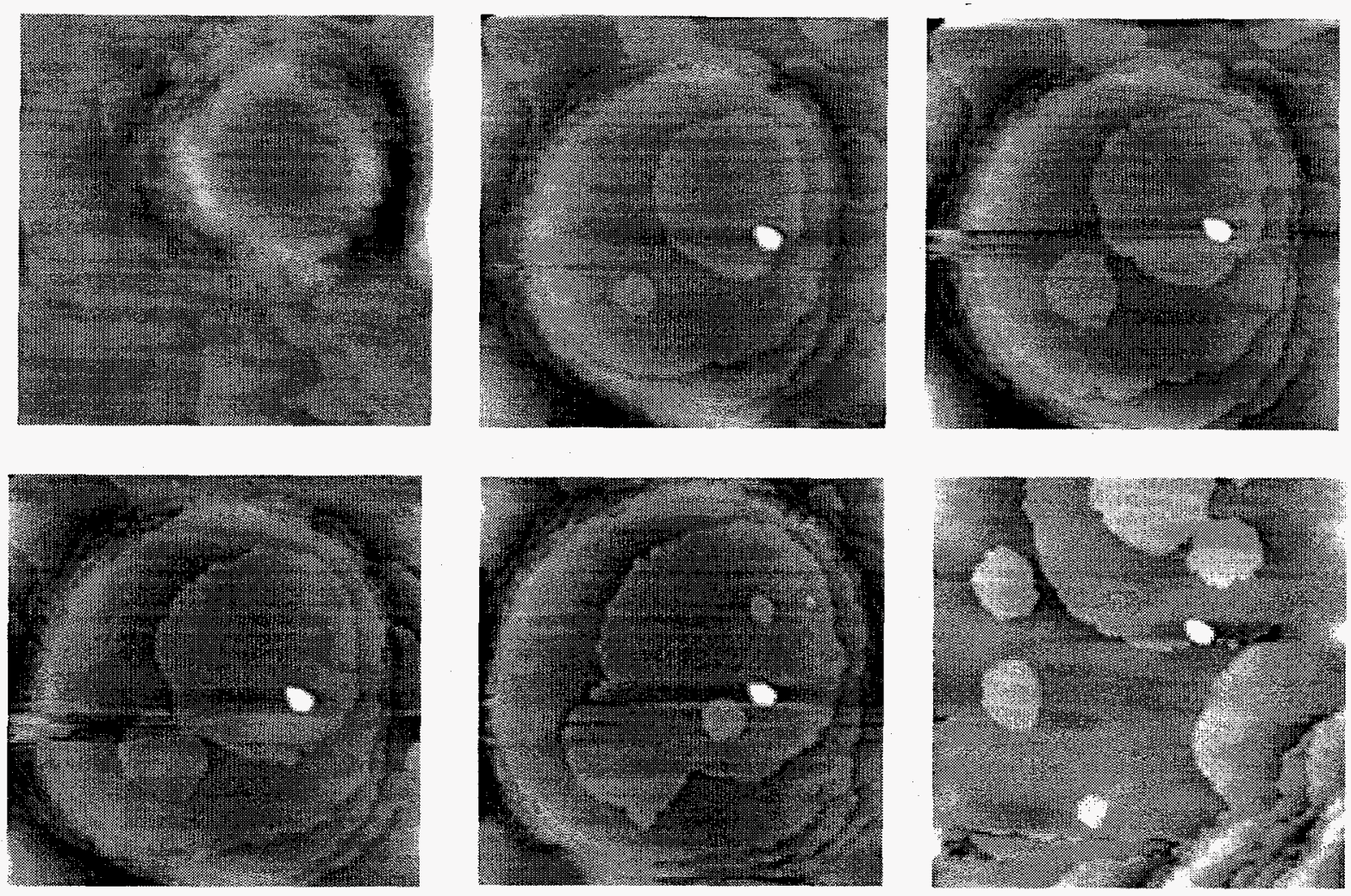

figure 5

DeYoreo/Land 

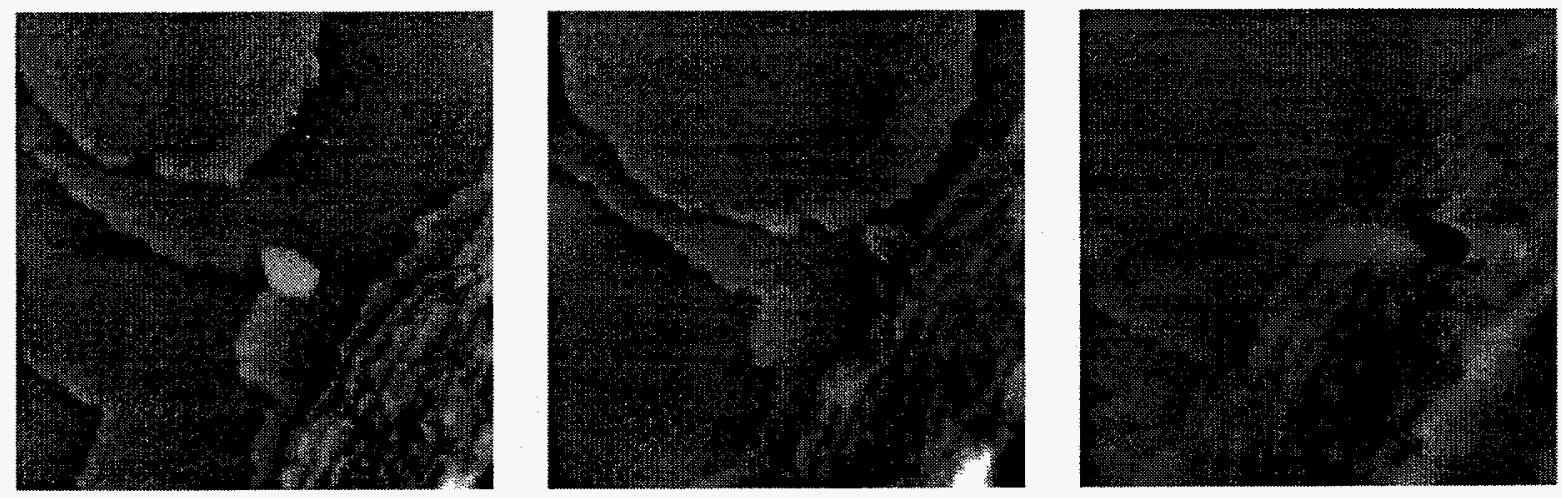

figure 6

DeYoreo/Land 


\section{DISCLAIMER}

This document was prepared as an acoount of work sponsored by an agency of the United States Government. Neither the United States Government nor the University of California nor any of their employees, makes any warranty, express or implied, or assumes any legal liability or responsibility for the accuracy, completeness, or usefulness of any information, apparatus, product, or process disclosed, or represents that its use would not infringe privately owned rights. Reference herein to any specific commercial product, process, or service by trade name, trademark, manufacturer, or otherwise, does not necessarily constitute or imply its endorsement, recommendation, or favoring by the United States Government or the University of California. The views and opinions of authors expressed herein do not necessarily state or reflect those of the United States Government or the University of California, and shall not be used for advertising or product endorsement purposes. 